\title{
On a certain new subclass of meromorphic close-to-convex functions
}

Huo Tang ${ }^{1,2^{*}}$, Guan-Tie Deng ${ }^{2}$ and Shu-Hai Li ${ }^{1}$

${ }^{*}$ Correspondence: thth2009@tom.com

'School of Mathematics and Statistics, Chifeng University, Chifeng, Inner Mongolia 024000, China

${ }^{2}$ School of Mathematical Sciences, Beijing Normal University, Beijing, 100875, China

\begin{abstract}
In this paper, we introduce and investigate a new subclass $M K^{(k)}(\beta, \gamma)$ of meromorphic close-to-convex functions. For functions belonging to the class $M K^{(k)}(\beta, \gamma)$, we obtain some coefficient inequalities and a distortion theorem. The results presented here would unify and extend some recent work of Wang et al. (Appl. Math. Lett. 25:454-460, 2012).
\end{abstract}

MSC: $30 C 45$

Keywords: analytic functions; meromorphic close-to-convex functions; coefficient inequality; distortion theorem; subordination

\section{Introduction}

Let $\Sigma$ be the class of functions $f$ of the form:

$$
f(z)=\frac{1}{z}+\sum_{n=1}^{\infty} a_{n} z^{n}
$$

which are analytic in the punctured open unit disk $U^{*}=\{z \in C: 0<|z|<1\}=U \backslash\{0\}$.

Let $P$ denote the class of functions $p$ given by

$$
p(z)=1+\sum_{n=1}^{\infty} p_{n} z^{n} \quad(z \in U)
$$

which are analytic and convex in $U$ and satisfy the condition $\Re(p(z))>0(z \in U)$.

A function $f \in \Sigma$ is said to be in the class $M S^{*}(\alpha)$ of meromorphic starlike functions of order $\alpha$ if it satisfies the inequality

$$
\Re\left(\frac{z f^{\prime}(z)}{f(z)}\right)<-\alpha \quad\left(z \in U^{*} ; 0 \leq \alpha<1\right)
$$

In addition, a function $f \in \Sigma$ is said to be in the class $M C$ of meromorphic close-to-convex functions if it satisfies the inequality

$$
\Re\left(\frac{z f^{\prime}(z)}{g(z)}\right)<0 \quad\left(z \in U^{*} ; g \in M S^{*}(0)=M S^{*}\right) .
$$

Recently, Srivastava et al. [1] (see also [2, 3]) introduced and studied the class $M S_{s}^{*}$ of meromorphic starlike functions with respect to symmetric points, which satisfies the con-

(C) 2013 Tang et al.; licensee Springer. This is an Open Access article distributed under the terms of the Creative Commons Attribution License (http://creativecommons.org/licenses/by/2.0), which permits unrestricted use, distribution, and reproduction in any medium, provided the original work is properly cited. 
dition

$$
\Re\left(\frac{z f^{\prime}(z)}{f(z)-f(-z)}\right)<0 \quad\left(z \in U^{*}\right) .
$$

More recently, Wang et al. [4] discussed a class $M K$ of meromorphic close-to-convex functions, that is, a function $f \in \Sigma$ is said to be in the class $M K$ if it satisfies the inequality

$$
\Re\left(\frac{f^{\prime}(z)}{g(z) g(-z)}\right)>0 \quad\left(z \in U^{*}\right)
$$

where $g \in M S^{*}\left(\frac{1}{2}\right)$.

Let $f(z)=z+a_{2} z^{2}+\cdots$ be analytic in $U$. If there exists a function $g \in S^{*}\left(\frac{1}{2}\right)$, such that

$$
\left|\frac{z^{2} f^{\prime}(z)}{g(z) g(-z)}+1\right|<\left|\frac{z^{2} f^{\prime}(z)}{g(z) g(-z)}-1+2 \gamma\right| \quad(z \in U)
$$

then we say that $f \in K_{s}(\gamma), 0 \leq \gamma<1$, where $S^{*}\left(\frac{1}{2}\right)$ denotes the usual class of starlike functions of order $1 / 2$. The function class $K_{s}(\gamma)$ was introduced and studied recently by Kowalczyk and Les-Bomba [5] (see also [6-9]).

For two functions $f$ and $g$ analytic in $U$, we say that the function $f(z)$ is subordinate to $g(z)$ in $U$, and we write $f(z) \prec g(z)(z \in U)$ if there exists a Schwarz function $w(z)$, analytic in $U$ with $w(0)=0$ and $|w(z)| \leq 1$, such that $f(z)=g(w(z))(z \in U)$. In particular, if the function $g$ is univalent in $U$, then we have $f(0)=g(0)$ and $f(U) \subset g(U)$ (see, for example, [10]).

Motivated essentially by the above mentioned function classes $M K$ and $K_{s}(\gamma)$, we now introduce a new class $M K^{(k)}(\beta, \gamma)$ of meromorphic functions.

Definition 1 Let $M K^{(k)}(\beta, \gamma)$ denote the class of functions in $\Sigma$ satisfying the inequality

$$
\left|\frac{z^{2-k} f^{\prime}(z)}{g_{k}(z)}+1\right|<\beta\left|\frac{z^{2-k} f^{\prime}(z)}{g_{k}(z)}+2 \gamma-1\right| \quad\left(z \in U^{*} ; 0<\beta \leq 1 ; 0 \leq \gamma<1\right)
$$

where $g \in M S^{*}\left(\frac{k-1}{k}\right), k \geq 1$, is a fixed positive integer and $g_{k}(z)$ is defined by the following equality:

$$
g_{k}(z)=\prod_{v=0}^{k-1} \varepsilon^{-v} g\left(\varepsilon^{v} z\right) \quad\left(\varepsilon^{k}=1\right) .
$$

We note that $M K^{(2)}(1,0)=M K$ (see [4]), so the class $M K^{(k)}(\beta, \gamma)$ is a generation of the class $M K$.

In this paper, we prove that the class $M K^{(k)}(\beta, \gamma)$ is a subclass of meromorphic closeto-convex functions. Moreover, we provide some coefficient inequalities and a distortion theorem for functions in the class $M K^{(k)}(\beta, \gamma)$. Our results unify and extend the corresponding results obtained by Wang et al. [4]. 


\section{Main results}

First of all, we give two meaningful conclusions about the class $M K^{(k)}(\beta, \gamma)$. The proof of Theorem 1 below is much akin to that of Theorem 1 in [11], so we choose to omit the details involved.

Theorem 1 A function $f \in M K^{(k)}(\beta, \gamma)$ if and only if there exists $g \in M S^{*}\left(\frac{k-1}{k}\right)$ such that

$$
-\frac{z^{2-k} f^{\prime}(z)}{g_{k}(z)} \prec \frac{1+(1-2 \gamma) \beta z}{1-\beta z} \quad\left(z \in U^{*}\right) .
$$

Remark 1 From Theorem 1, we know that

$$
\Re\left(\frac{z^{2-k} f^{\prime}(z)}{g_{k}(z)}\right)<0 \quad\left(z \in U^{*}\right),
$$

because of $\Re\left(\frac{1+(1-2 \gamma) \beta z}{1-\beta z}\right)>0\left(z \in U^{*}\right)$.

Lemma 1 Let $\varphi_{i} \in M S^{*}\left(\alpha_{i}\right)$, where $0 \leq \alpha_{i}<1(i=0,1, \ldots, k-1)$. Then for $k-1 \leq \sum_{i=0}^{k-1} \alpha_{i}<k$, we have

$$
z^{k-1} \prod_{i=0}^{k-1} \varphi_{i}(z) \in M S^{*}\left(\sum_{i=0}^{k-1} \alpha_{i}-(k-1)\right) .
$$

Proof Since $\varphi_{i} \in M S^{*}\left(\alpha_{i}\right)(i=0,1, \ldots, k-1)$, by the definition of meromorphic starlike functions, we have

$$
\Re\left(\frac{z \varphi_{0}^{\prime}(z)}{\varphi_{0}(z)}\right)<-\alpha_{0}, \quad \Re\left(\frac{z \varphi_{1}^{\prime}(z)}{\varphi_{1}(z)}\right)<-\alpha_{1}, \quad \ldots, \quad \Re\left(\frac{z \varphi_{k-1}^{\prime}(z)}{\varphi_{k-1}(z)}\right)<-\alpha_{k-1} .
$$

We now let

$$
F(z)=z^{k-1} \varphi_{0}(z) \varphi_{1}(z) \cdots \varphi_{k-1}(z) .
$$

Differentiating (2.4) with respect to $z$ logarithmically, we easily get

$$
\frac{z F^{\prime}(z)}{F(z)}=\frac{z \varphi_{0}^{\prime}(z)}{\varphi_{0}(z)}+\frac{z \varphi_{1}^{\prime}(z)}{\varphi_{1}(z)}+\cdots+\frac{z \varphi_{k-1}^{\prime}(z)}{\varphi_{k-1}(z)}+(k-1) .
$$

From (2.5) together with (2.3), we obtain

$$
\begin{aligned}
\Re\left(\frac{z F^{\prime}(z)}{F(z)}\right) & =\Re\left(\frac{z \varphi_{0}^{\prime}(z)}{\varphi_{0}(z)}\right)+\Re\left(\frac{z \varphi_{1}^{\prime}(z)}{\varphi_{1}(z)}\right)+\cdots+\Re\left(\frac{z \varphi_{k-1}^{\prime}(z)}{\varphi_{k-1}(z)}\right)+(k-1) \\
& <-\sum_{i=0}^{k-1} \alpha_{i}+(k-1)=-\left(\sum_{i=0}^{k-1} \alpha_{i}-(k-1)\right)
\end{aligned}
$$

by noting that $0 \leq \sum_{i=0}^{k-1} \alpha_{i}-(k-1)<1$, which implies that

$$
F(z)=z^{k-1} \prod_{i=0}^{k-1} \varphi_{i}(z) \in M S^{*}\left(\sum_{i=0}^{k-1} \alpha_{i}-(k-1)\right) .
$$

The proof of Lemma 1 is thus completed. 
Theorem 2 Let $g(z)=\frac{1}{z}+\sum_{n=1}^{\infty} b_{n} z^{n} \in M S^{*}\left(\frac{k-1}{k}\right)$, then $z^{k-1} g_{k}(z) \in M S^{*}$.

Proof From (1.4), we know

$$
\begin{aligned}
z^{k-1} g_{k}(z) & =z^{k-1} \prod_{v=0}^{k-1} \varepsilon^{-v} g\left(\varepsilon^{v} z\right)=z^{k-1} \prod_{v=0}^{k-1} \varepsilon^{-v}\left[\frac{1}{\varepsilon^{v} z}+\sum_{n=1}^{\infty} b_{n}\left(\varepsilon^{v} z\right)^{n}\right] \\
& =z^{k-1} \prod_{v=0}^{k-1}\left[\frac{1}{\varepsilon^{2 v} z}+\sum_{n=1}^{\infty} b_{n} \varepsilon^{(n-1) v} z^{n}\right]
\end{aligned}
$$

Since $g(z)=\frac{1}{z}+\sum_{n=1}^{\infty} b_{n} z^{n} \in M S^{*}\left(\frac{k-1}{k}\right)$, by Lemma 1 and (2.6), we can easily get the assertion of Theorem 2 .

Remark 2 From Theorem 2 and the inequality (2.2), we see that if $f \in \operatorname{MK}^{(k)}(\beta, \gamma)$, then $f(z)$ is a meromorphic close-to-convex function. So, $M K^{(k)}(\beta, \gamma)$ is a subclass of the class $M C$ of meromorphic close-to-convex functions.

Next, we give some coefficient inequalities for functions belonging to the class $M K^{(k)}(\beta, \gamma)$.

Theorem 3 Let $f(z)=\frac{1}{z}+\sum_{n=1}^{\infty} a_{n} z^{n}$ and $g(z)=\frac{1}{z}+\sum_{n=1}^{\infty} b_{n} z^{n}$ be analytic in $U^{*}$. If for $0<\beta \leq 1$ and $0 \leq \gamma<1$, we have

$$
\sum_{n=1}^{\infty} n(1+\beta)\left|a_{n}\right|+\sum_{n=1}^{\infty}(\beta|1-2 \gamma|+1)\left|B_{n}\right| \leq 2 \beta(1-\gamma)
$$

where the coefficients $B_{n}(n=1,2, \ldots)$ are given by (2.9), then $f \in M K^{(k)}(\beta, \gamma)$.

Proof Suppose that

$$
G_{k}(z)=z^{k-1} g_{k}(z)
$$

By Theorem 2, we know that $G_{k} \in M S^{*}$. Hence, equality (2.6) can be written as

$$
G_{k}(z)=z^{k-1} g_{k}(z)=\frac{1}{z}+\sum_{n=1}^{\infty} B_{n} z^{n} \in M S^{*}
$$

To prove $f \in M K^{(k)}(\beta, \gamma)$, it suffices to show that

$$
\left|\frac{\frac{z f^{\prime}(z)}{G_{k}(z)}+1}{\frac{z f^{\prime}(z)}{G_{k}(z)}+2 \gamma-1}\right|<\beta
$$

where $G_{k}$ is given by (2.8). From (2.7), we know that

$$
\beta(2-2 \gamma)-\sum_{n=1}^{\infty} n \beta\left|a_{n}\right|-\sum_{n=1}^{\infty} \beta|1-2 \gamma|\left|B_{n}\right| \geq \sum_{n=1}^{\infty} n\left|a_{n}\right|+\sum_{n=1}^{\infty}\left|B_{n}\right|>0 .
$$


Now, by the maximum modulus principle, we deduce from (1.1), (2.9) and (2.10) that

$$
\begin{aligned}
\left|\frac{\frac{z f^{\prime}(z)}{G_{k}(z)}+1}{\frac{z f^{\prime}(z)}{G_{k}(z)}+2 \gamma-1}\right| & =\left|\frac{\sum_{n=1}^{\infty} n a_{n} z^{n+1}+\sum_{n=1}^{\infty} B_{n} z^{n+1}}{\sum_{n=1}^{\infty} n a_{n} z^{n+1}-\sum_{n=1}^{\infty}(1-2 \gamma) B_{n} z^{n+1}-(2-2 \gamma)}\right| \\
& <\frac{\sum_{n=1}^{\infty} n\left|a_{n}\right|+\sum_{n=1}^{\infty}\left|B_{n}\right|}{(2-2 \gamma)-\sum_{n=1}^{\infty} n\left|a_{n}\right|-\sum_{n=1}^{\infty}|1-2 \gamma|\left|B_{n}\right|} \leq \beta .
\end{aligned}
$$

This evidently completes the proof of Theorem 3 .

Theorem 4 Let $f(z)=\frac{1}{z}+\sum_{n=1}^{\infty} a_{n} z^{n} \in M K^{(k)}(\beta, \gamma)$. Then

$$
\begin{aligned}
& 1+\sum_{n=1}^{\infty} \frac{n\left(1-\beta e^{i \theta}\right)}{2(1-\gamma) \beta e^{i \theta}} a_{n} z^{n+1}+\sum_{n=1}^{\infty} \frac{1+(1-2 \gamma) \beta e^{i \theta}}{2(1-\gamma) \beta e^{i \theta}} B_{n} z^{n+1} \neq 0 \\
& \left(z \in U^{*} ; 0<\theta<2 \pi\right),
\end{aligned}
$$

where the coefficients $B_{n}(n=1,2, \ldots)$ are given by (2.9).

Proof Suppose that $f \in M K^{(k)}(\beta, \gamma)$. Then we know that

$$
-\frac{z f^{\prime}(z)}{G_{k}(z)} \not \frac{1+(1-2 \gamma) \beta e^{i \theta}}{1-\beta e^{i \theta}} \quad\left(z \in U^{*} ; 0<\theta<2 \pi\right)
$$

where $G_{k}$ is given by (2.8). After a simple computation, the inequality (2.2) is equivalent to

$$
z f^{\prime}(z)\left(1-\beta e^{i \theta}\right)+G_{k}(z)\left(1+(1-2 \gamma) \beta e^{i \theta}\right) \neq 0 \quad\left(z \in U^{*} ; 0<\theta<2 \pi\right) .
$$

By substituting (1.1) and (2.9) into (2.13), we obtain the desired assertion (2.11) of Theorem 4.

Finally, we provide the following distortion theorem for the considered class of functions $M K^{(k)}(\beta, \gamma)$.

Theorem 5 Iff $\in M K^{(k)}(\beta, \gamma)$, then

$$
\begin{aligned}
\frac{(1-r)^{2}(1-(1-2 \gamma) \beta r)}{r^{2}(1+\beta r)} & \leq\left|f^{\prime}(z)\right| \\
& \leq \frac{(1+r)^{2}(1+(1-2 \gamma) \beta r)}{r^{2}(1-\beta r)} \quad(|z|=r ; 0<r<1) .
\end{aligned}
$$

Proof If $f \in M K^{(k)}(\beta, \gamma)$, then there exists a function $g \in M S^{*}\left(\frac{k-1}{k}\right)$ such that (1.3) holds true. It follows from Theorem 2 that the function $G_{k}$ given by (2.8) is a meromorphic starlike function. Hence, we have (see [12])

$$
\frac{(1-r)^{2}}{r} \leq\left|G_{k}(z)\right| \leq \frac{(1+r)^{2}}{r} \quad(|z|=r ; 0<r<1) .
$$


Let us define $p(z)$ by

$$
-\frac{z f^{\prime}(z)}{G_{k}(z)}=p(z) \quad\left(z \in U^{*}\right)
$$

where

$$
p(z) \prec \frac{1+(1-2 \gamma) \beta z}{1-\beta z} .
$$

Then, by using a similar method as in [13, p.105], we have

$$
\frac{1-(1-2 \gamma) \beta r}{1+\beta r} \leq|p(z)| \leq \frac{1+(1-2 \gamma) \beta r}{1-\beta r} \quad(|z|=r ; 0<r<1) .
$$

Thus, from (2.15), (2.16) and (2.17), we readily get the inequality (2.14). The proof of Theorem 5 is thus completed.

\section{Competing interests}

The authors declare that they have no competing interests.

\section{Authors' contributions}

All authors jointly worked on the results and they read and approved the final manuscript.

\section{Acknowledgements}

The present investigation was partly supported by the Natural Science Foundation of China under Grant 11271045, the Higher School Doctoral Foundation of China under Grant 20100003110004 and the Natural Science Foundation of Inner Mongolia of China under Grant 2010MS0117. The authors are thankful to the referees for their careful reading and making some helpful comments which have essentially improved the presentation of this paper.

Received: 23 September 2012 Accepted: 14 March 2013 Published: 10 April 2013

\section{References}

1. Srivastava, HM, Yang, D-G, Xu, N: Some subclasses of meromorphically multivalent functions associated with a linear operator. Appl. Math. Comput. 195, 11-23 (2008)

2. Chandrashekar, R, Ali, RM, Lee, SK, Ravichandran, V: Convolutions of meromorphic multivalent functions with respect to $n$-ply points and symmetric conjugate points. Appl. Math. Comput. 218, 723-728 (2011)

3. Wang, Z-G, Jiang, Y-P, Srivastava, HM: Some subclasses of meromorphically multivalent functions associated with the generalized hypergeometric function. Comput. Math. Appl. 57, 571-586 (2009)

4. Wang, Z-G, Sun, Y, Xu, N: Some properties of certain meromorphic close-to-convex functions. Appl. Math. Lett. 25 454-460 (2012)

5. Kowalczyk, J, Les-Bomba, E: On a subclass of close-to-convex functions. Appl. Math. Lett. 23, 1147-1151 (2010)

6. Seker, B: On certain new subclass of close-to-convex functions. Appl. Math. Comput. 218, 1041-1045 (2011)

7. Xu, Q-H, Srivastava, HM, Li, Z: A certain subclass of analytic and close-to-convex functions. Appl. Math. Lett. 24, 396-401 (2011)

8. Cho, NE, Kwon, OS, Ravichandran, V: Coefficient, distortion and growth inequalities for certain close-to-convex functions. J. Inequal. Appl. 2011, 1-7 (2011)

9. Goswami, P, Bulut, S, Bulboaca, T: Certain properties of a new subclass of close-to-convex functions. Arab. J. Math (2012). doi:10.1007/s40065-012-0029-y

10. Miller, SS, Mocanu, PT: Differential Subordination: Theory and Applications. Series on Monographs and Textbooks in Pure and Applied Mathematics, vol. 225. Dekker, New York (2000)

11. Wang, Z-G, Gao, C-Y, Yuan, S-M: On certain subclass of close-to-convex functions. Acta Math. Acad. Paedagog. Nyházi. 22, 171-177 (2006)

12. Pommerenke, C: On meromorphic starlike functions. Pac. J. Math. 13, 221-235 (1963)

13. Goodman, AW: Univalent Functions, vol. 1. Polygonal Publishing House, Washington (1983)

doi:10.1186/1029-242X-2013-164

Cite this article as: Tang et al.: On a certain new subclass of meromorphic close-to-convex functions. Journal of Inequalities and Applications 2013 2013:164. 